\title{
TWO-POINT FORMULAE OF EULER TYPE
}

\author{
M. MATIĆ ${ }^{1}$, C. E. M. PEARCE ${ }^{2}$ and J. PEČARIĆ ${ }^{3}$
}

(Received 7 October, 1998)

\begin{abstract}
An analysis is made of quadrature via two-point formulae when the integrand is Lipschitz or of bounded variation. The error estimates are shown to be as good as those found in recent studies using Simpson (three-point) formulae.
\end{abstract}

\section{Introduction and preliminaries}

The simplest quadrature rule of open type is based on the well-known midpoint formula

$$
\int_{a}^{b} f(t) \mathrm{d} t=(b-a) f\left(\frac{a+b}{2}\right)+\frac{(b-a)^{3}}{24} f^{\prime \prime}(\xi),
$$

where $a<\xi<b$ (see [3, p. 71]). Another quadrature rule of this type is based on the two-point formula

$$
\int_{a}^{b} f(t) \mathrm{d} t=\frac{b-a}{2}\left[f\left(\frac{2 a+b}{3}\right)+f\left(\frac{a+2 b}{3}\right)\right]+\frac{(b-a)^{3}}{36} f^{\prime \prime}(\eta),
$$

where $a<\eta<b$ (see [3, p. 70]). Both formulae apply provided $f:[a, b] \rightarrow \mathbf{R}$ is in the class $C^{2}[a, b]$.

For a convex function $f \in C^{2}[a, b]$ we have $f^{\prime \prime}(\xi) \geq 0$, so a simple consequence of (1.1) for such functions is the Hadamard inequality

$$
\frac{1}{b-a} \int_{a}^{b} f(t) \mathrm{d} t \geq f\left(\frac{a+b}{2}\right) \text {. }
$$

\footnotetext{
'FESB, Mathematics Department, University of Split, R. Boškovića bb, 21000 Split, Croatia; e-mail: mmatic@fesb.hr.

${ }^{2}$ Applied Mathematics Department, The University of Adelaide, Adelaide SA 5005, Australia; e-mail: cpearce@maths.adelaide.edu.au.

${ }^{3}$ Applied Mathematics Department, The University of Adelaide, Adelaide SA 5005, Australia: e-mail: jpecaric@maths.adelaide.edu.au.
}

(C) Australian Mathematical Society 2002, Serial-fee code 1446-1811/02 
By the same argument, (1.2) yields

$$
\frac{1}{b-a} \int_{a}^{b} f(t) \mathrm{d} t \geq \frac{1}{2}\left[f\left(\frac{2 a+b}{3}\right)+f\left(\frac{a+2 b}{3}\right)\right]
$$

for any convex function $f \in C^{2}[a, b]$.

Inequality (1.4) is tighter than (1.3) for $f$ convex, since

$$
\frac{1}{2}\left[f\left(\frac{2 a+b}{3}\right)+f\left(\frac{a+2 b}{3}\right)\right] \geq f\left(\frac{1}{2} \cdot \frac{2 a+b}{3}+\frac{1}{2} \cdot \frac{a+2 b}{3}\right)=f\left(\frac{a+b}{2}\right) .
$$

However, we can obtain (1.4) by using (1.3) on subintervals. The latter inequality provides

$$
\begin{aligned}
\int_{a}^{b} f(t) \mathrm{d} t & =\int_{a}^{(a+b) / 2} f(t) \mathrm{d} t+\int_{(a+b) / 2}^{b} f(t) \mathrm{d} t \\
& \geq \frac{b-a}{2}\left[f\left(\frac{3 a+b}{4}\right)+f\left(\frac{a+3 b}{4}\right)\right] .
\end{aligned}
$$

On the other hand, a convex function $f:[a, b] \rightarrow \mathbf{R}$ satisfies

$$
f(x+z)-f(x) \leq f(y+z)-f(y)
$$

whenever $x, y$ and $z$ are such that $x, x+z, y, y+z \in[a, b]$ with $x \leq y$ and $z \geq 0$ (see [11, p. 3]). In particular, the choices $x=(3 a+b) / 4, y=(a+2 b) / 3$ and $z=(b-a) / 12$ yield

$$
f\left(\frac{2 a+b}{3}\right)-f\left(\frac{3 a+b}{4}\right) \leq f\left(\frac{a+3 b}{4}\right)-f\left(\frac{a+2 b}{3}\right),
$$

that is,

$$
f\left(\frac{2 a+b}{3}\right)+f\left(\frac{a+2 b}{3}\right) \leq f\left(\frac{3 a+b}{4}\right)+f\left(\frac{a+3 b}{4}\right) .
$$

Combining this with (1.5) supplies (1.4).

Midpoint formulae of Euler type, based on (1.1), were treated recently in [4]. In this paper we consider similar results related to the two-point formula (1.2).

The fundamental ingredients in our analysis are the same, namely the two identities

$$
f(x)=\frac{1}{b-a} \int_{a}^{b} f(t) \mathrm{d} t+T_{n}(x)+R_{n}^{1}(x)
$$

and

$$
f(x)=\frac{1}{b-a} \int_{a}^{b} f(t) \mathrm{d} t+T_{n-1}(x)+R_{n}^{2}(x),
$$


which may conveniently be referred to as the extended Euler formulae and which were established recently in [5]. Here $T_{0}(x)=0$ and

$$
T_{m}(x)=\sum_{k=1}^{m} \frac{(b-a)^{k-1}}{k !} B_{k}\left(\frac{x-a}{b-a}\right)\left[f^{(k-1)}(b)-f^{(k-1)}(a)\right]
$$

for $m \geq 1$, while

$$
R_{n}^{1}(x)=-\frac{(b-a)^{n-1}}{n !} \int_{[a, b]} B_{n}^{*}\left(\frac{x-t}{b-a}\right) \mathrm{d} f^{(n-1)}(t)
$$

and

$$
R_{n}^{2}(x)=-\frac{(b-a)^{n-1}}{n !} \int_{[a, b]}\left[B_{n}^{*}\left(\frac{x-t}{b-a}\right)-B_{n}\left(\frac{x-a}{b-a}\right)\right] \mathrm{d} f^{(n-1)}(t) .
$$

We write $\int_{[a, b]} g(t) \mathrm{d} \varphi(t)$ here, as throughout the paper, to denote the RiemannStieltjes integral of $g$ with respect to a function $\varphi:[a, b] \rightarrow \mathbf{R}$ of bounded variation and $\int_{a}^{b} g(t) \mathrm{d} t$ for the Riemann integral. The identities (1.6) and (1.7) extend the well-known formula for the expansion of a function in terms of Bernoulli polynomials $\left[10\right.$, p. 17]. They hold for every function $f:[a, b] \rightarrow \mathbf{R}$ such that $f^{(n-1)}$ is continuous and of bounded variation on $[a, b]$ for some $n \geq 1$ and for every $x \in[a, b]$. The functions $B_{k}(t)$ are the Bernoulli polynomials, $B_{k}=B_{k}(0)$ the Bernoulli numbers and $B_{k}^{*}(t)(k \geq 0)$ are functions of period 1 related to the Bernoulli polynomials via

$$
\begin{aligned}
B_{k}^{*}(t) & =B_{k}(t), & & \text { for } 0 \leq t<1, \\
B_{k}^{*}(t+1) & =B_{k}^{*}(t), & & \text { for } t \in \mathbf{R} .
\end{aligned}
$$

The Bernoulli polynomials $B_{k}(t)(k \geq 0)$ are uniquely determined by the identities

$$
B_{k}^{\prime}(t)=k B_{k-1}(t), \quad k \geq 1 ; B_{0}(t)=1
$$

and

$$
B_{k}(t+1)-B_{k}(t)=k t^{k-1}, \quad k \geq 0 .
$$

For further details on the Bernoulli polynomials and the Bernoulli numbers, see for example [1] or [2]. We have

$$
B_{0}(t)=1, \quad B_{1}(t)=t-1 / 2, \quad B_{2}(t)=t^{2}-t+1 / 6, \quad B_{3}(t)=t^{3}-3 t^{2} / 2+t / 2,
$$

so that $B_{0}^{*}(t)=1$ and $B_{1}^{*}(t)$ has a jump of -1 at each integer. From (1.10) it follows that $B_{k}(1)=B_{k}(0)=B_{k}$ for $k \geq 2$, so that $B_{k}^{*}(t)$ is continuous for $k \geq 2$. Moreover, using (1.9) we get

$$
B_{k}^{* \prime}=k B_{k-1}^{*}(t), \quad k \geq 1
$$

and this holds for every $t \in \mathbf{R}$ when $k \geq 3$, and for every $t \in \mathbf{R} \backslash \mathbf{Z}$ when $k=1,2$. 
As in [4], our analysis hangs on detailed properties of the Bernoulli polynomials. The analysis is effected via two families $\left(F_{k}\right)_{k \geq 1}$ and $\left(G_{k}\right)_{k \geq 1}$ of auxiliary functions. The basic idea of the two-point approach is outlined in Section 2 and centres on two two-point formulae. In Section 3 we develop the requisite results for the auxiliary functions and in Section 4 use these to determine error estimates when integrals are approximated by our two-point formulae. We consider integrands which are either of bounded variation or possess a Lipschitz property. We find that the error estimates for our current two-point procedures are as good as those obtained recently for threepoint (Simpson) procedures (see [6-9]). Finally in Section 5 we make corresponding estimates when the domain of integration is given a general uniform partition and the two-point formulae are repeated for quadrature.

\section{Generalisations of the two-point formula}

For $k \geq 1$, define the functions $G_{k}(t)$ and $F_{k}(t)$ by

$$
G_{k}(t):=B_{k}^{*}(1 / 3-t)+B_{k}^{*}(2 / 3-t), \quad t \in \mathbf{R}
$$

and

$$
F_{k}(t):=G_{k}(t)-\tilde{B}_{k}, \quad t \in \mathbf{R},
$$

where

$$
\tilde{B}_{k}:=G_{k}(0)=B_{k}(1 / 3)+B_{k}(2 / 3), \quad k \geq 1 .
$$

The functions $G_{k}(t)$ and $F_{k}(t)$ are of period 1 and continuous for $k \geq 2$ and so are determined by their behaviour on $[0,1]$. This we investigate in the next section.

Let $f:[a, b] \rightarrow \mathbf{R}$ be such that $f^{(n-1)}$ exists on $[a, b]$ for some $n \geq 1$. We introduce the notation

$$
M(a, b):=\frac{b-a}{2}\left[f\left(\frac{2 a+b}{3}\right)+f\left(\frac{a+2 b}{3}\right)\right] .
$$

Further, define

$$
\tilde{T}_{0}(a, b):=0
$$

and

$$
\tilde{T}_{m}(a, b):=\frac{b-a}{2}\left[T_{m}\left(\frac{2 a+b}{3}\right)+T_{m}\left(\frac{a+2 b}{3}\right)\right]
$$

for $1 \leq m \leq n$, where $T_{m}(x)$ is given by (1.8). Then

$$
\tilde{T}_{m}(a, b)=\frac{1}{2} \sum_{k=1}^{m} \frac{(b-a)^{k}}{k !} \tilde{B}_{k}\left[f^{(k-1)}(b)-f^{(k-1)}(a)\right] .
$$


In the theorem below we establish two formulae which we term two-point formulae of Euler type and which play a key role in this paper.

THEOREM 1. Let $f:[a, b] \rightarrow \mathbf{R}$ be such that $f^{(n-1)}$ is continuous and of bounded variation on $[a, b]$ for some $n \geq 1$. Then

$$
\int_{a}^{b} f(t) \mathrm{d} t=M(a, b)-\tilde{T}_{n}(a, b)+\tilde{R}_{n}^{1}(a, b),
$$

where

Also

$$
\tilde{R}_{n}^{1}(a, b)=\frac{(b-a)^{n}}{2(n !)} \int_{[a, b]} G_{n}\left(\frac{t-a}{b-a}\right) \mathrm{d} f^{(n-1)}(t) .
$$

where

$$
\int_{a}^{b} f(t) \mathrm{d} t=M(a, b)-\tilde{T}_{n-1}(a, b)+\tilde{R}_{n}^{2}(a, b),
$$

$$
\tilde{R}_{n}^{2}(a, b)=\frac{(b-a)^{n}}{2(n !)} \int_{[a, b]} F_{n}\left(\frac{t-a}{b-a}\right) \mathrm{d} f^{(n-1)}(t) .
$$

Proof. Put $x=(2 a+b) / 3,(a+2 b) / 3$ in (1.6), multiply the two resultant formulae by $(b-a) / 2$ and add. This produces (2.3). Formula (2.4) is obtained from (1.7) by the same procedure.

REMARK 1. Suppose that $f:[a, b] \rightarrow \mathbf{R}$ is such that $f^{(n)}$ exists and is integrable on $[a, b]$ for some $n \geq 1$. In this case (2.3) holds with

while (2.4) holds with

$$
\tilde{R}_{n}^{1}(a, b)=\frac{(b-a)^{n}}{2(n !)} \int_{a}^{b} G_{n}\left(\frac{t-a}{b-a}\right) f^{(n)}(t) \mathrm{d} t
$$

$$
\tilde{R}_{n}^{2}(a, b)=\frac{(b-a)^{n}}{2(n !)} \int_{a}^{b} F_{n}\left(\frac{t-a}{b-a}\right) f^{(n)}(t) \mathrm{d} t .
$$

By direct calculation we get $\tilde{B}_{1}=0, \tilde{B}_{2}=-1 / 9, \tilde{B}_{3}=0$. This implies, by (2.2), that

$$
\tilde{T}_{0}(a, b)=\tilde{T}_{1}(a, b)=0, \quad \tilde{T}_{2}(a, b)=\tilde{T}_{3}(a, b)=-\frac{(b-a)^{2}}{36}\left[f^{\prime}(b)-f^{\prime}(a)\right]
$$

Also

$$
G_{1}(t)=F_{1}(t)= \begin{cases}-2 t, & 0 \leq t \leq 1 / 3 \\ -2 t+1, & 1 / 3<t \leq 2 / 3 \\ -2 t+2, & 2 / 3<t \leq 1\end{cases}
$$




$$
\begin{gathered}
G_{2}(t)= \begin{cases}2 t^{2}-1 / 9, & 0 \leq t \leq 1 / 3 \\
2 t^{2}-2 t+5 / 9, & 1 / 3<t \leq 2 / 3 \\
2 t^{2}-4 t+17 / 9, & 2 / 3<t \leq 1\end{cases} \\
F_{2}(t)= \begin{cases}2 t^{2}, & 0 \leq t \leq 1 / 3 \\
2 t^{2}-2 t+2 / 3, & 1 / 3<t \leq 2 / 3 \\
2 t^{2}-4 t+2, & 2 / 3<t \leq 1\end{cases}
\end{gathered}
$$

and

$$
F_{3}(t)=G_{3}(t)= \begin{cases}-2 t^{3}+t / 3, & 0 \leq t \leq 1 / 3 \\ -2 t^{3}+3 t^{2}-5 t / 3+1 / 3, & 1 / 3<t \leq 2 / 3 \\ -2 t^{3}+6 t^{2}-17 t / 3+5 / 3, & 2 / 3<t \leq 1\end{cases}
$$

Applying (2.4) with $n=1,2$ yields the identities

$$
\begin{aligned}
\int_{a}^{b} f(t) \mathrm{d} t-M(a, b) & =\frac{b-a}{2} \int_{[a, b]} F_{1}\left(\frac{t-a}{b-a}\right) \mathrm{d} f(t) \\
& =\frac{(b-a)^{2}}{4} \int_{[a, b]} F_{2}\left(\frac{t-a}{b-a}\right) \mathrm{d} f^{\prime}(t) .
\end{aligned}
$$

Similarly, (2.4) with $n=3,4$ generates the identities

$$
\begin{aligned}
& \int_{a}^{b} f(t) \mathrm{d} t-M(a, b)-\frac{(b-a)^{2}}{36}\left[f^{\prime}(b)-f^{\prime}(a)\right] \\
& \quad=\frac{(b-a)^{3}}{12} \int_{[a, b]} F_{3}\left(\frac{t-a}{b-a}\right) \mathrm{d} f^{\prime \prime}(t)=\frac{(b-a)^{4}}{48} \int_{[a, b]} F_{4}\left(\frac{t-a}{b-a}\right) \mathrm{d} f^{\prime \prime \prime}(t) .
\end{aligned}
$$

\section{The auxiliary functions}

To proceed to error estimates, we need some properties of the functions $G_{k}(t)$ and $F_{k}(t)$. As noted earlier, it is enough to know these on $[0,1]$.

The Bernoulli polynomials of even order are symmetric and those of odd order skew-symmetric about $1 / 2$, that is,

$$
B_{k}(1-t)=(-1)^{k} B_{k}(t), \quad 0 \leq t \leq 1, \quad k \geq 1
$$

(see [1, 23.1.8]). Setting $t=1 / 3$ gives $B_{k}(2 / 3)=(-1)^{k} B_{k}(1 / 3)$, so that

$$
\tilde{B}_{k}=B_{k}(1 / 3)+B_{k}(2 / 3)=\left[1+(-1)^{k}\right] B_{k}(1 / 3) \quad(k \geq 1),
$$

which implies $\tilde{B}_{2 k-1}=0, \tilde{B}_{2 k}=2 B_{2 k}(1 / 3)(k \geq 1)$. Also

$$
B_{2 k}(1 / 3)=-2^{-1}\left(1-3^{1-2 k}\right) B_{2 k}, \quad k \geq 1,
$$


(see $[1,23.1 .23])$, which gives

$$
\tilde{B}_{2 k-1}=0, \quad \tilde{B}_{2 k}=-\left(1-3^{1-2 k}\right) B_{2 k}, \quad k \geq 1 .
$$

Now by (3.3) we have

$$
F_{2 k-1}(t)=G_{2 k-1}(t), \quad k \geq 1
$$

and

$$
F_{2 k}(t)=G_{2 k}(t)+\left(1-3^{1-2 k}\right) B_{2 k}, \quad k \geq 1
$$

Further, the points 0 and 1 are zeros of $F_{n}(t)$, that is, $F_{n}(0)=F_{n}(1)=0(n \geq 1)$. As we shall see below, they are the only zeros of $F_{n}(t)$ for $n=2 k(k \geq 1)$. Also, using (3.1) again, we get $G_{n}(1 / 2)=B_{n}(5 / 6)+B_{n}(1 / 6)=\left[(-1)^{n}+1\right] B_{n}(1 / 6)$. Hence for $n=2 k-1(k \geq 1)$ we have $F_{2 k-1}(1 / 2)=G_{2 k-1}(1 / 2)=0$.

We shall see that $0,1 / 2$ and 1 are the only zeros of $F_{2 k-1}(t)=G_{2 k-1}(t)$ for $k \geq 1$. Also note that for $n=2 k(k \geq 1)$ we have

$$
G_{2 k}(0)=G_{2 k}(1)=\tilde{B}_{2 k}=-\left(1-3^{1-2 k}\right) B_{2 k} .
$$

Using $[1,23.1 .24] B_{2 k}(1 / 6)=B_{2 k}(5 / 6)=2^{-1}\left(1-2^{1-2 k}\right)\left(1-3^{1-2 k}\right) B_{2 k}, k \geq 1$, we get

$$
G_{2 k}(1 / 2)=2 B_{2 k}(1 / 6)=\left(1-2^{1-2 k}\right)\left(1-3^{1-2 k}\right) B_{2 k} \quad(k \geq 1)
$$

while $F_{2 k}(1 / 2)=G_{2 k}(1 / 2)-\tilde{B}_{2 k}=2\left(1-2^{-2 k}\right)\left(1-3^{1-2 k}\right) B_{2 k}, k \geq 1$.

LEMMA 1. For $n \geq 2$ we have $G_{n}(1-t)=(-1)^{n} G_{n}(t)$ and $F_{n}(1-t)=(-1)^{n} F_{n}(t)$, $0 \leq t \leq 1$

Proof. Since $B_{n}^{*}(t)$ is of period 1 and continuous for $n \geq 2$, we have for $n \geq 2$ and $0 \leq t \leq 1$ that

$$
\begin{aligned}
G_{n}(t)= & B_{n}^{*}(1 / 3-t)+B_{n}^{*}(2 / 3-t) \\
= & \begin{cases}B_{n}(1 / 3-t)+B_{n}(2 / 3-t), & 0 \leq t \leq 1 / 3 \\
B_{n}(4 / 3-t)+B_{n}(2 / 3-t), & 1 / 3<t \leq 2 / 3 \\
B_{n}(4 / 3-t)+B_{n}(5 / 3-t), & 2 / 3<t \leq 1\end{cases}
\end{aligned}
$$

and

$$
\begin{aligned}
G_{n}(1-t)= & B_{n}^{*}(-2 / 3+t)+B_{n}^{*}(-1 / 3+t) \\
& = \begin{cases}B_{n}(1 / 3+t)+B_{n}(2 / 3+t), & 0 \leq t<1 / 3 \\
B_{n}(1 / 3+t)+B_{n}(-1 / 3+t), & 1 / 3 \leq t<2 / 3 \\
B_{n}(-2 / 3+t)+B_{n}(-1 / 3+t), & 2 / 3 \leq t \leq 1\end{cases}
\end{aligned}
$$


Further, using (3.1) we get

$$
G_{n}(1-t)=(-1)^{n} \times \begin{cases}B_{n}(1 / 3-t)+B_{n}(2 / 3-t), & 0 \leq t<1 / 3 ; \\ B_{n}(4 / 3-t)+B_{n}(2 / 3-t), & 1 / 3 \leq t<2 / 3 \\ B_{n}(4 / 3-t)+B_{n}(5 / 3-t), & 2 / 3 \leq t \leq 1 .\end{cases}
$$

Since $G_{n}(t)$ is continuous for $n \geq 2, G_{n}(1-t)=(-1)^{n} G_{n}(t), 0 \leq t \leq 1$, which proves the first identity. Further, we have $F_{n}(t)=G_{n}(t)-G_{n}(0)$ and $(-1)^{n} G_{n}(0)=$ $G_{n}(0)$, since $G_{2 k-1}(0)=0$, so that

$$
F_{n}(1-t)=G_{n}(1-t)-G_{n}(0)=(-1)^{n}\left[G_{n}(t)-G_{n}(0)\right]=(-1)^{n} F_{n}(t),
$$

which proves the second identity.

Note that the identities established in Lemma 1 are valid for $n=1$ too except at the points $1 / 3$ and $2 / 3$ of discontinuity of $F_{1}(t)=G_{1}(t)$.

LEMMA 2. For $k \geq 2$ the function $G_{2 k-1}(t)$ has no zeros in the interval $(0,1 / 2)$. The sign of this function is determined by $(-1)^{k} G_{2 k-1}(t)>0,0<t<1 / 2$.

Proof. For $k=2, G_{3}(t)$ is given by (2.9) and we have $G_{3}(t)>0(0<t<1 / 2)$, so our assertion is true for $k=2$. Now, assume that $k \geq 3$. Then $2 k-1 \geq 5$ and $G_{2 k-1}(t)$ is continuous and twice differentiable. Using (1.12) we get

$$
G_{2 k-1}^{\prime}(t)=-(2 k-1) G_{2 k-2}(t)
$$

and

$$
G_{2 k-1}^{\prime \prime}(t)=(2 k-1)(2 k-2) G_{2 k-3}(t) .
$$

We know that 0 and $1 / 2$ are zeros of $G_{2 k-1}(t)$. Suppose that some $\alpha \in(0,1 / 2)$ is also a zero of $G_{2 k-1}(t)$. Then the derivative $G_{2 k-1}^{\prime}(t)$ must have at least one zero $\beta_{1} \in(0, \alpha)$ and at least one zero $\beta_{2} \in(\alpha, 1 / 2)$. Therefore $G_{2 k-1}^{\prime \prime}(t)$ must have at least one zero inside $\left(\beta_{1}, \beta_{2}\right)$. Thus, from the assumption that $G_{2 k-1}(t)$ has a zero inside $(0,1 / 2)$, it follows from (3.8) that $G_{2 k-3}(t)$ also has a zero inside this interval, and so by induction $G_{3}(t)$ has a zero on $(0,1 / 2)$, which we have seen not to be the case. Hence $G_{2 k-1}(t)$ cannot have a zero on $(0,1 / 2)$.

To determine the sign of $G_{2 k-1}(t)$, note that

$$
G_{2 k-1}(1 / 3)=B_{2 k-1}(0)+B_{2 k-1}(1 / 3)=B_{2 k-1}(1 / 3) .
$$

We have from $[1,23.1 .14]$ that $(-1)^{k} B_{2 k-1}(t)>0(0<t<1 / 2)$, which implies

$$
(-1)^{k} G_{2 k-1}(1 / 3)=(-1)^{k} B_{2 k-1}(1 / 3)>0 .
$$

Consequently $(-1)^{k} G_{2 k-1}(t)>0(0<t<1 / 2)$. 
COROLlaRY 1. For $k \geq 2$ the functions $(-1)^{k-1} F_{2 k}(t)$ and $(-1)^{k-1} G_{2 k}(t)$ are strictly increasing on $(0,1 / 2)$ and strictly decreasing on $(1 / 2,1)$. Consequently, 0 and 1 are the only zeros of $F_{2 k}(t)$ in $[0,1]$ and

$$
\max _{t \in[0,1]}\left|F_{2 k}(t)\right|=2\left(1-2^{-2 k}\right)\left(1-3^{1-2 k}\right)\left|B_{2 k}\right|, \quad k \geq 2 .
$$

Also $\max _{t \in[0,1]}\left|G_{2 k}(t)\right|=\left(1-3^{1-2 k}\right)\left|B_{2 k}\right|, k \geq 2$.

PROOF. Using (1.12) we get $\left[(-1)^{k-1} F_{2 k}(t)\right]^{\prime}=\left[(-1)^{k-1} G_{2 k}(t)\right]^{\prime}=2 k(-1)^{k} G_{2 k-1}(t)$ and $(-1)^{k} G_{2 k-1}(t)>0$ for $0<t<1 / 2$ by Lemma 2 . Thus $(-1)^{k-1} F_{2 k}(t)$ and $(-1)^{k-1} G_{2 k}(t)$ are strictly increasing on $(0,1 / 2)$. Also by Lemma $1, F_{2 k}(1-t)=$ $F_{2 k}(t)$ and $G_{2 k}(1-t)=G_{2 k}(t)(0 \leq t \leq 1)$, which implies that $(-1)^{k-1} F_{2 k}(t)$ and $(-1)^{k-1} G_{2 k}(t)$ are strictly decreasing on $(1 / 2,1)$. Further, $F_{2 k}(0)=F_{2 k}(1)=0$, which implies that $\left|F_{2 k}(t)\right|$ achieves its maximum at $t=1 / 2$, that is,

$$
\max _{i \in[0,1]}\left|F_{2 k}(t)\right|=\left|F_{2 k}(1 / 2)\right|=2\left(1-2^{-2 k}\right)\left(1-3^{1-2 k}\right)\left|B_{2 k}\right| .
$$

Also

$$
\begin{aligned}
\max _{t \in[0,1]}\left|G_{2 k}(t)\right| & =\max \left\{\left|G_{2 k}(0)\right|,\left|G_{2 k}(1 / 2)\right|\right\} \\
& =\max \left\{\left(1-3^{1-2 k}\right)\left|B_{2 k}\right|,\left(1-2^{1-2 k}\right)\left(1-3^{1-2 k}\right)\left|B_{2 k}\right|\right\} \\
& =\left(1-3^{1-2 k}\right)\left|B_{2 k}\right|
\end{aligned}
$$

which completes the proof.

COROLLARY 2. If $k \geq 2$,

$$
\int_{0}^{1}\left|F_{2 k-1}(t)\right| \mathrm{d} t=\int_{0}^{1}\left|G_{2 k-1}(t)\right| \mathrm{d} t=\frac{2}{k}\left(1-2^{-2 k}\right)\left(1-3^{1-2 k}\right)\left|B_{2 k}\right| .
$$

Also

$$
\begin{aligned}
& \int_{0}^{1}\left|F_{2 k}(t)\right| \mathrm{d} t=\left|\tilde{B}_{2 k}\right|=\left(1-3^{1-2 k}\right)\left|B_{2 k}\right| \quad \text { and } \\
& \int_{0}^{1}\left|G_{2 k}(t)\right| \mathrm{d} t \leq 2\left|\tilde{B}_{2 k}\right|=2\left(1-3^{1-2 k}\right)\left|B_{2 k}\right| .
\end{aligned}
$$

PROOF. Using (1.12) we get

$$
G_{m}^{\prime}(t)=-m G_{m-1}(t), \quad m \geq 3 .
$$

By (3.4) we have $\int_{0}^{1}\left|F_{2 k-1}(t)\right| \mathrm{d} t=\int_{0}^{1}\left|G_{2 k-1}(t)\right| \mathrm{d} t$. By Lemmas 1 and 2 and (3.9) we get

$$
\int_{0}^{1}\left|G_{2 k-1}(t)\right| \mathrm{d} t=2\left|\int_{0}^{1 / 2} G_{2 k-1}(t) \mathrm{d} t\right|=\frac{1}{k}\left|G_{2 k}(1 / 2)-G_{2 k}(0)\right| .
$$


The first assertion follows from (3.7) and (3.6).

From (3.5), (3.9) and the periodicity of $G_{m}$ for $m \geq 2$, we have

$$
\int_{0}^{1} F_{2 k}(s) \mathrm{d} s=\left(1-3^{1-2 k}\right) B_{2 k}=-\tilde{B}_{2 k},
$$

by (3.3), which leads to the second assertion. Finally, we use (3.5) again and the triangle inequality to obtain

$$
\int_{0}^{1}\left|G_{2 k}(t)\right| \mathrm{d} t=\int_{0}^{1}\left|F_{2 k}(t)+\tilde{B}_{2 k}\right| \mathrm{d} t \leq \int_{0}^{1}\left|F_{2 k}(t)\right| \mathrm{d} t+\left|\tilde{B}_{2 k}\right|=2\left|\tilde{B}_{2 k}\right|,
$$

which proves the third assertion.

\section{Two-point formula error estimates}

In this section we use the two-point formulae of Euler type established in Theorem 1 to prove a number of inequalities for various classes of functions.

THEOREM 2. Let $f:[a, b] \rightarrow \mathbf{R}$ be such that $f^{(n-1)}$ is an L-Lipschitzian function on $[a, b]$ for some $n \geq 1$. Then

$$
\left|\int_{a}^{b} f(t) \mathrm{d} t-M(a, b)+\tilde{T}_{n-1}(a, b)\right| \leq \frac{(b-a)^{n+1}}{2(n !)} \int_{0}^{1}\left|F_{n}(t)\right| \mathrm{d} t \cdot L .
$$

Also

$$
\left|\int_{a}^{b} f(t) \mathrm{d} t-M(a, b)+\tilde{T}_{n}(a, b)\right| \leq \frac{(b-a)^{n+1}}{2(n !)} \int_{0}^{1}\left|G_{n}(t)\right| \mathrm{d} t \cdot L .
$$

PROOF. For any integrable function $\Phi:[a, b] \rightarrow \mathbf{R}$ we have

$$
\left|\int_{[a, b]} \Phi(t) \mathrm{d} f^{(n-1)}(t)\right| \leq \int_{a}^{b}|\Phi(t)| \mathrm{d} t \cdot L,
$$

since $f^{(n-1)}$ is $L$-Lipschitzian. Applying (4.3) with $\Phi(t)=F_{n}((t-a) /(b-a))$ gives

$$
\begin{aligned}
\left|\frac{(b-a)^{n}}{2(n !)} \int_{[a, b]} F_{n}\left(\frac{t-a}{b-a}\right) \mathrm{d} f^{(n-1)}(t)\right| & \leq \frac{(b-a)^{n}}{2(n !)} \int_{a}^{b}\left|F_{n}\left(\frac{t-a}{b-a}\right)\right| \mathrm{d} t \cdot L \\
& =\frac{(b-a)^{n+1}}{2(n !)} \int_{0}^{1}\left|F_{n}(t)\right| \mathrm{d} t \cdot L .
\end{aligned}
$$

Applying the above inequality, we get (4.1) from (2.4). Similarly, we can apply (4.3) with $\Phi(t)=G_{n}((t-a) /(b-a))$ and then use (2.3) to obtain (4.2). 
COROLLARY 3. Let $f:[a, b] \rightarrow \mathbf{R}$.

If $f$ is L-Lipschitzian, then $\left|\int_{a}^{b} f(t) \mathrm{d} t-M(a, b)\right| \leq(5 / 36)(b-a)^{2} \cdot L$.

If $f^{\prime}$ is L-Lipschitzian, then $\left|\int_{a}^{b} f(t) \mathrm{d} t-M(a, b)\right| \leq(1 / 36)(b-a)^{3} \cdot L$.

If $f^{\prime \prime}$ is L-Lipschitzian, then

$$
\left|\int_{a}^{b} f(t) \mathrm{d} t-M(a, b)-\frac{(b-a)^{2}}{36}\left[f^{\prime}(b)-f^{\prime}(a)\right]\right| \leq \frac{13}{5184}(b-a)^{4} \cdot L .
$$

If $f^{\prime \prime \prime}$ is L-Lipschitzian, then

$$
\left|\int_{a}^{b} f(t) \mathrm{d} t-M(a, b)-\frac{(b-a)^{2}}{36}\left[f^{\prime}(b)-f^{\prime}(a)\right]\right| \leq \frac{13}{19440}(b-a)^{5} \cdot L .
$$

PROOF. Using (2.6) and (2.7) we get $\int_{0}^{1}\left|F_{1}(t)\right| \mathrm{d} t=5 / 18$ and $\int_{0}^{1}\left|F_{2}(t)\right| \mathrm{d} t=1 / 9$, respectively. Therefore, using (2.5) and (2.1) and applying (4.1) with $n=1$ and $n=2$, we get the first and second inequalities, respectively. By Corollary 2, $\int_{0}^{1}\left|F_{3}(t)\right| \mathrm{d} t=13 / 432$ and $\int_{0}^{1}\left|F_{4}(t)\right| \mathrm{d} t=13 / 405$. The third inequality follows from (4.1) with $n=3$ and (2.5), while the fourth follows from (4.1) with $n=4$ and (2.5).

REMARK 2. For a function $f$ which is $L$-Lipschitzian on $[a, b]$,

$$
\left|\int_{a}^{b} f(t) \mathrm{d} t-\frac{b-a}{3}\left[\frac{f(a)+f(b)}{2}+2 f\left(\frac{a+b}{2}\right)\right]\right| \leq \frac{5}{36}(b-a)^{2} \cdot L
$$

(see [7] and [9]). This inequality is related to Simpson's quadrature formula and gives an error estimate for an $L$-Lipschitzian function on $[a, b]$. This may be compared with the first inequality

$$
\left|\int_{a}^{b} f(t) \mathrm{d} t-\frac{b-a}{2}\left[f\left(\frac{2 a+b}{3}\right)+f\left(\frac{a+2 b}{3}\right)\right]\right| \leq \frac{5}{36}(b-a)^{2} \cdot L
$$

in Corollary 3. We see that, for this class of function, we have the same error estimate for the two-point quadrature rule as for Simpson's rule. However Simpson's rule requires the evaluation of $f$ at three points, while the two-point rule requires evaluation at two points only. Error estimates applying with the repeated use of these formulae for a finite interval consisting of $v$ subintervals will also agree. In that context the Simpson scheme will involve evaluations at $2 v+1$ points and our present procedure $2 v$ points.

COROLlaRY 4. Let $f:[a, b] \rightarrow \mathbf{R}$ be such that $f^{(n-1)}$ is L-Lipschitzian on $[a, b]$ for some $n \geq 2$. Set $D_{0}(a, b):=0$ and for any integer $r$ such that $1 \leq r \leq n / 2$ define

$$
D_{r}(a, b):=-\frac{1}{2} \sum_{i=1}^{r} \frac{(b-a)^{2 i}}{(2 i) !}\left(1-3^{1-2 i}\right) B_{2 i}\left[f^{(2 i-1)}(b)-f^{(2 i-1)}(a)\right] .
$$


If $n=2 k-1(k \geq 2)$, then

$\left|\int_{a}^{b} f(t) \mathrm{d} t-M(a, b)+D_{k-1}(a, b)\right| \leq \frac{(b-a)^{2 k}}{(2 k) !} 2\left(1-2^{-2 k}\right)\left(1-3^{1-2 k}\right)\left|B_{2 k}\right| \cdot L$.

If $n=2 k(k \geq 2)$, then

and

$$
\left|\int_{a}^{b} f(t) \mathrm{d} t-M(a, b)+D_{k-1}(a, b)\right| \leq \frac{(b-a)^{2 k+1}}{2[(2 k) !]}\left(1-3^{1-2 k}\right)\left|B_{2 k}\right| \cdot L
$$

$$
\left|\int_{a}^{b} f(t) \mathrm{d} t-M(a, b)+D_{k}(a, b)\right| \leq \frac{(b-a)^{2 k+1}}{(2 k) !}\left(1-3^{1-2 k}\right)\left|B_{2 k}\right| \cdot L .
$$

Proof. For $n=2 k-1$ we have by (4.5) that $\tilde{T}_{n-1}(a, b)=D_{k-1}(a, b)$. Thus the first inequality follows from Corollary 2 and (4.1). Moreover, for $m \geq 2$ we have that

$$
\begin{aligned}
\tilde{T}_{m}(a, b) & =\frac{1}{2} \sum_{k=1}^{[m / 2]} \frac{(b-a)^{2 k}}{(2 k) !} \tilde{B}_{2 k}\left[f^{(2 k-1)}(b)-f^{(2 k-1)}(a)\right] \\
& =-\frac{1}{2} \sum_{k=1}^{[m / 2]} \frac{(b-a)^{2 k}}{(2 k) !}\left(1-3^{1-2 k}\right) B_{2 k}\left[f^{(2 k-1)}(b)-f^{(2 k-1)}(a)\right],
\end{aligned}
$$

where $[x]$ denotes the greatest integer less than or equal to $x$. Hence we have for $n=2 k$ that $\tilde{T}_{n-1}(a, b)=D_{k-1}(a, b)$ and $\tilde{T}_{n}(a, b)=D_{k}(a, b)$. The second inequality follows from Corollary 2 and (4.1) and the third from Corollary 2 and (4.2).

REMARK 3. Suppose that $f:[a, b] \rightarrow \mathbf{R}$ is such that $f^{(n)}$ exists and is bounded on $[a, b]$, for some $n \geq 1$. In this case we have for all $t, s \in[a, b]$ that

$$
\left|f^{(n-1)}(t)-f^{(n-1)}(s)\right| \leq\left\|f^{(n)}\right\|_{\infty} \cdot|t-s|,
$$

so that $f^{(n-1)}$ is $\left\|f^{(n)}\right\|_{\infty}$-Lipschitzian on $[a, b]$. Therefore the inequalities established in Theorem 2 hold with $L=\left\|f^{(n)}\right\|_{\infty}$. Consequently, under appropriate assumptions on $f$, the inequalities from Corollary 3 hold with $L=\left\|f^{\prime}\right\|_{\infty},\left\|f^{\prime \prime}\right\|_{\infty},\left\|f^{\prime \prime \prime}\right\|_{\infty}$ and $\left\|f^{\prime \prime \prime \prime}\right\|_{\infty}$, respectively. A similar observation can be made for the results of Corollary 4.

In the next theorem and subsequently we denote by $V_{a}^{b}(f)$ the total variation of $f$ on $[a, b]$.

THEOREM 3. Let $f:[a, b] \rightarrow \mathbf{R}$ be such that $f^{(n-1)}$ is continuous and of bounded variation on $[a, b]$ for some $n \geq 1$. Then

$$
\left|\int_{a}^{b} f(t) \mathrm{d} t-M(a, b)+\tilde{T}_{n-1}(a, b)\right| \leq \frac{(b-a)^{n}}{2(n !)} \max _{t \in[0,1]}\left|F_{n}(t)\right| \cdot V_{a}^{b}\left(f^{(n-1)}\right)
$$


and

$$
\left|\int_{a}^{b} f(t) \mathrm{d} t-M(a, b)+\tilde{T}_{n}(a, b)\right| \leq \frac{(b-a)^{n}}{2(n !)} \max _{t \in[0,1]}\left|G_{n}(t)\right| \cdot V_{a}^{b}\left(f^{(n-1)}\right)
$$

Proof. If $\Phi:[a, b] \rightarrow \mathbf{R}$ is bounded on $[a, b]$ and the Riemann-Stieltjes integral $\int_{[a, b]} \Phi(t) \mathrm{d} f^{(n-1)}(t)$ exists, then

$$
\left|\int_{[a, b]} \Phi(t) \mathrm{d} f^{(n-1)}(t)\right| \leq \max _{t \in[a, b]}|\Phi(t)| \cdot V_{a}^{b}\left(f^{(n-1)}\right)
$$

We apply the estimate (4.8) to $\Phi(t)=F_{n}((t-a) /(b-a))$ to obtain

$$
\begin{aligned}
\left|\frac{(b-a)^{n}}{2(n !)} \int_{[a, b]} F_{n}\left(\frac{t-a}{b-a}\right) \mathrm{d} f^{(n-1)}(t)\right| & \leq \frac{(b-a)^{n}}{2(n !)} \max _{t \in[a, b]}\left|F_{n}\left(\frac{t-a}{b-a}\right)\right| \cdot V_{a}^{b}\left(f^{(n-1)}\right) \\
& =\frac{(b-a)^{n+1}}{2(n !)} \max _{t \in[0,1]}\left|F_{n}(t)\right| \cdot V_{a}^{b}\left(f^{(n-1)}\right) .
\end{aligned}
$$

We now use the above inequality and (2.4) to obtain (4.6). In the same way, we apply the estimate (4.8) to $\Phi(t)=G_{n}((t-a) /(b-a))$, and then use (2.3) to obtain (4.7).

COROLlaRY 5. Let $f:[a, b] \rightarrow \mathbf{R}$.

If $f$ is continuous and of bounded variation on $[a, b]$, then

$$
\left|\int_{a}^{b} f(t) \mathrm{d} t-M(a, b)\right| \leq \frac{b-a}{3} \cdot V_{a}^{b}(f)
$$

If $f^{\prime}$ is continuous and of bounded variation on $[a, b]$, then

$$
\left|\int_{a}^{b} f(t) \mathrm{d} t-M(a, b)\right| \leq \frac{1}{18}(b-a)^{2} \cdot V_{a}^{b}\left(f^{\prime}\right)
$$

If $f^{\prime \prime}$ is continuous and of bounded variation on $[a, b]$, then

$$
\left|\int_{a}^{b} f(t) \mathrm{d} t-M(a, b)-\frac{(b-a)^{2}}{36}\left[f^{\prime}(b)-f^{\prime}(a)\right]\right| \leq \frac{\sqrt{2}}{324}(b-a)^{3} \cdot V_{a}^{b}\left(f^{\prime \prime}\right)
$$

If $f^{\prime \prime \prime}$ is continuous and of bounded variation on $[a, b]$, then

$$
\left|\int_{a}^{b} f(t) \mathrm{d} t-M(a, b)-\frac{(b-a)^{2}}{36}\left[f^{\prime}(b)-f^{\prime}(a)\right]\right| \leq \frac{13}{10368}(b-a)^{4} \cdot V_{a}^{b}\left(f^{\prime \prime \prime}\right)
$$


PROOF. From the explicit expressions (2.6), (2.8) and (2.9), we get

$$
\max _{t \in[0,1]}\left|F_{1}(t)\right|=-F_{1}(1 / 3)=2 / 3, \quad \max _{t \in[0,1]}\left|F_{2}(t)\right|=F_{2}(1 / 3)=2 / 9
$$

and

$$
\max _{t \in[0,1]}\left|F_{3}(t)\right|=F_{3}\left(\frac{1}{3 \sqrt{2}}\right)=\frac{\sqrt{2}}{27},
$$

respectively. Therefore, using (2.5) and applying (4.6) with $n=1,2,3$, we get respectively the first, second and third inequalities. Further, by Corollary 1 ,

$$
\max _{t \in[0,1]}\left|F_{4}(t)\right|=13 / 216 .
$$

The fourth inequality follows from (4.6) with $n=4$ and (2.5).

REMARK 4. It has been established in [8] (see also [9]) that

$$
\left|\int_{a}^{b} f(t) \mathrm{d} t-\frac{b-a}{3}\left[\frac{f(a)+f(b)}{2}+2 f\left(\frac{a+b}{2}\right)\right]\right| \leq \frac{b-a}{3} \cdot V_{a}^{b}(f) .
$$

This inequality is related to Simpson's quadrature formula and gives the error estimate for a function of bounded variation on $[a, b]$. This may be compared with the first inequality

$$
\left|\int_{a}^{b} f(t) \mathrm{d} t-\frac{b-a}{2}\left[f\left(\frac{2 a+b}{3}\right)+f\left(\frac{a+2 b}{3}\right)\right]\right| \leq \frac{b-a}{3} \cdot V_{a}^{b}(f)
$$

in Corollary 5. The comparison in Remark 2 also applies here.

COROLLARY 6. Let $f:[a, b] \rightarrow \mathbf{R}$ be such that $f^{(n-1)}$ is continuous and of bounded variation on $[a, b]$ for some $n \geq 2$. Define $D_{r}(a, b)(r \geq 0)$ as in Corollary 4. If $n=2 k-1(k \geq 2)$, then

$$
\left|\int_{a}^{b} f(t) \mathrm{d} t-M(a, b)+D_{k-1}(a, b)\right| \leq \frac{(b-a)^{2 k-1}}{2[(2 k-1) !]} \max _{t \in[0,1]}\left|F_{2 k-1}(t)\right| \cdot V_{a}^{b}\left(f^{(2 k-2)}\right) .
$$

If $n=2 k(k \geq 2)$, then

$$
\left|\int_{a}^{b} f(t) \mathrm{d} t-M(a, b)+D_{k-1}(a, b)\right| \leq \frac{(b-a)^{2 k}}{(2 k) !}\left(1-2^{-2 k}\right)\left(1-3^{1-2 k}\right)\left|B_{2 k}\right| \cdot V_{a}^{b}\left(f^{(2 k-1)}\right)
$$

and

$$
\left|\int_{a}^{b} f(t) \mathrm{d} t-M(a, b)+D_{k}(a, b)\right| \leq \frac{(b-a)^{2 k}}{2[(2 k) !]}\left(1-3^{1-2 k}\right)\left|B_{2 k}\right| \cdot V_{a}^{b}\left(f^{(2 k-1)}\right) .
$$


PROOF. The argument is similar to that used in the proof of Corollary 4. We apply Theorem 3 and use the formulae established in Corollary 1.

REMARK 5. Suppose that $f:[a, b] \rightarrow \mathbf{R}$ is such that $f^{(n)} \in L_{1}[a, b]$ for some $n \geq 1$. In this case $f^{(n-1)}$ is continuous and of bounded variation on $[a, b]$ and we have $V_{a}^{b}\left(f^{(n-1)}\right)=\int_{a}^{b}\left|f^{(n)}(t)\right| \mathbf{d} t=\left\|f^{(n)}\right\|_{1}$. Therefore the inequalities established in Theorem 3 hold with $\left\|f^{(n)}\right\|_{1}$ in place of $V_{a}^{b}\left(f^{(n-1)}\right)$. A similar observation can be made for the results of Corollaries 5 and 6.

THEOREM 4. Suppose $(p, q)$ is a pair of conjugate exponents, which we may specify as $1<p, q<\infty$ with $p^{-1}+q^{-1}=1$ or $p=\infty, q=1$, and let $f:[a, b] \rightarrow \mathbf{R}$ be such that $f^{(n)} \in L_{p}[a, b]$ for some $n \geq 1$. Then

$$
\left|\int_{a}^{b} f(t) \mathrm{d} t-M(a, b)+\tilde{T}_{n-1}(a, b)\right| \leq K(n, p)(b-a)^{n+1 / q} \cdot\left\|f^{(n)}\right\|_{p},
$$

where $K(n, p)=(1 / 2(n !))\left(\int_{0}^{1}\left|F_{n}(t)\right|^{q} \mathrm{~d} t\right)^{1 / q}$. Also

$$
\left|\int_{a}^{b} f(t) \mathrm{d} t-M(a, b)+\tilde{T}_{n}(a, b)\right| \leq K^{*}(n, p)(b-a)^{n+1 / q} \cdot\left\|f^{(n)}\right\|_{p},
$$

where $K^{*}(n, p)=(1 / 2(n !))\left(\int_{0}^{1}\left|G_{n}(t)\right|^{q} \mathrm{~d} t\right)^{1 / q}$.

PROOF. By the Hölder inequality, we have

$$
\begin{aligned}
& \left|\frac{(b-a)^{n}}{2(n !)} \int_{a}^{b} F_{n}\left(\frac{t-a}{b-a}\right) f^{(n)}(t) \mathrm{d} t\right| \\
& \quad \leq \frac{(b-a)^{n}}{2(n !)}\left[\int_{a}^{b}\left|F_{n}\left(\frac{t-a}{b-a}\right)\right|^{q} \mathrm{~d} t\right]^{1 / q}\left\|f^{(n)}\right\|_{p} \\
& \quad=\frac{(b-a)^{n+1 / q}}{2(n !)}\left[\int_{0}^{1}\left|F_{n}(t)\right|^{q} \mathrm{~d} t\right]^{1 / q}\left\|f^{(n)}\right\|_{p}=K(n, p)(b-a)^{n+1 / q}\left\|f^{(n)}\right\|_{p} .
\end{aligned}
$$

From this inequality, we get the estimate (4.6) from (2.4) and Remark 1. In the same way we get the estimate (4.10) from (2.3).

REMARK 6. For $p=\infty$ we have

$$
K(n, \infty)=\frac{1}{2(n !)} \int_{0}^{1}\left|F_{n}(t)\right| \mathrm{d} t \quad \text { and } \quad K^{*}(n, \infty)=\frac{1}{2(n !)} \int_{0}^{1}\left|G_{n}(t)\right| \mathrm{d} t
$$


The results established in Theorem 4 for $p=\infty$ coincide with those of Theorem 2 with $L=\left\|f^{(n)}\right\|_{\infty}$. Moreover, by Remark 3 and Corollary 3 , we have for $n=1,2$ that $\left|\int_{a}^{b} f(t) \mathrm{d} t-M(a, b)\right| \leq K(n, \infty)(b-a)^{n+1}\left\|f^{(n)}\right\|_{\infty}$, while for $n=3,4$ we have

$$
\left|\int_{a}^{b} f(t) \mathrm{d} t-M(a, b)-\frac{(b-a)^{2}}{36}\left[f^{\prime}(b)-f^{\prime}(a)\right]\right| \leq K(n, \infty)(b-a)^{n+1}\left\|f^{(n)}\right\|_{\infty},
$$

where $K(1, \infty)=5 / 36, K(2, \infty)=1 / 36, K(3, \infty)=13 / 5184, K(4, \infty)=13 / 19440$. Further, by Remark 3 and Corollary 4, we have for $k \geq 2$ that

$$
\begin{aligned}
& \left|\int_{a}^{b} f(t) \mathrm{d} t-M(a, b)+D_{k-1}(a, b)\right| \leq K(2 k-1, \infty)(b-a)^{2 k} \cdot\left\|f^{(2 k-1)}\right\|_{\infty}, \\
& \left|\int_{a}^{b} f(t) \mathrm{d} t-M(a, b)+D_{k-1}(a, b)\right| \leq K(2 k, \infty)(b-a)^{2 k+1} \cdot\left\|f^{(2 k)}\right\|_{\infty}
\end{aligned}
$$

and

$$
\left|\int_{a}^{b} f(t) \mathrm{d} t-M(a, b)+D_{k}(a, b)\right| \leq K^{*}(2 k, \infty)(b-a)^{2 k+1} \cdot\left\|f^{(2 k)}\right\|_{\infty},
$$

where

$$
\begin{gathered}
K(2 k-1, \infty)=\frac{2\left(1-2^{-2 k}\right)\left(1-3^{1-2 k}\right)}{(2 k) !}\left|B_{2 k}\right| \\
K(2 k, \infty)=\frac{1-3^{1-2 k}}{2[(2 k) !]}\left|B_{2 k}\right| \quad \text { and } \quad K^{*}(2 k, \infty) \leq \frac{1-3^{1-2 k}}{(2 k) !}\left|B_{2 k}\right|
\end{gathered}
$$

REMARK 7. For $p=1$ define

$$
K(n, 1):=\frac{1}{2(n !)} \max _{t \in[0,1]}\left|F_{n}(t)\right| \text { and } K^{*}(n, 1):=\frac{1}{2(n !)} \max _{t \in[0,1]}\left|G_{n}(t)\right| .
$$

Then, using Remark 5 and Theorem 3, we can extend the results established in Theorem 4 to the pair $p=1, q=\infty$. Thus if we set $1 / q=0$, then (4.9) and (4.10) hold for $p=1$. Also, by Remark 5 and Corollary 5, we have for $n=1,2$ that $\left|\int_{a}^{b} f(t) \mathrm{d} t-M(a, b)\right| \leq K(n, 1)(b-a)^{n}\left\|f^{(n)}\right\|_{1}$, while for $n=3,4$ we have

$$
\left|\int_{a}^{b} f(t) \mathrm{d} t-M(a, b)-\frac{(b-a)^{2}}{36}\left[f^{\prime}(b)-f^{\prime}(a)\right]\right| \leq K(n, 1)(b-a)^{n}\left\|f^{(n)}\right\|_{1},
$$

where $K(1,1)=1 / 3, K(2,1)=1 / 18, K(3,1)=\sqrt{2} / 324, K(4,1)=31 / 10368$. Further, by Remark 5 and Corollary 6 , for $k \geq 2$ we have

$$
\left|\int_{a}^{b} f(t) \mathrm{d} t-M(a, b)+D_{k-1}(a, b)\right| \leq K(2 k-1,1)(b-a)^{2 k-1}\left\|f^{(2 k-1)}\right\|_{1},
$$




$$
\left|\int_{a}^{b} f(t) \mathrm{d} t-M(a, b)+D_{k-1}(a, b)\right| \leq K(2 k, 1)(b-a)^{2 k}\left\|f^{(2 k)}\right\|_{1}
$$

and

$$
\left|\int_{a}^{b} f(t) \mathrm{d} t-M(a, b)+D_{k}(a, b)\right| \leq K^{*}(2 k, 1)(b-a)^{2 k}\left\|f^{(2 k)}\right\|_{1},
$$

where

$$
\begin{gathered}
K(2 k-1,1)=\frac{1}{2[(2 k-1) !]} \max _{t \in[0,1]}\left|F_{2 k-1}(t)\right| \\
K(2 k, 1)=\frac{\left(1-2^{-2 k}\right)\left(1-3^{1-2 k}\right)}{(2 k) !}\left|B_{2 k}\right| \text { and } K^{*}(2 k, 1)=\frac{1-3^{1-2 k}}{2[(2 k) !]}\left|B_{2 k}\right| .
\end{gathered}
$$

REMARK 8. For $1<p \leq \infty$ we can easily determine

$$
K(1, p)=\frac{1}{6}\left[\frac{2^{q+1}+1}{3(q+1)}\right]^{1 / q}
$$

so that for $n=1$ Theorem 4 yields

$$
\begin{aligned}
& \left|\int_{a}^{b} f(t) \mathrm{d} t-\frac{b-a}{2}\left[f\left(\frac{2 a+b}{3}\right)+f\left(\frac{a+2 b}{3}\right)\right]\right| \\
& \quad \leq \frac{1}{6}\left[\frac{2^{q+1}+1}{3(q+1)}\right]^{1 / q}(b-a)^{1+1 / q}\left\|f^{\prime}\right\|_{p} .
\end{aligned}
$$

This may be compared with the similar inequality proved in [6] (see also [9]), related to Simpson's rule

$$
\begin{aligned}
& \left|\int_{a}^{b} f(t) \mathrm{d} t-\frac{b-a}{3}\left[\frac{f(a)+f(b)}{2}+2 f\left(\frac{a+b}{2}\right)\right]\right| \\
& \leq \frac{1}{6}\left[\frac{2^{q+1}+1}{3(q+1)}\right]^{1 / q}(b-a)^{1+1 / q}\left\|f^{\prime}\right\|_{p} .
\end{aligned}
$$

The comparison in Remark 2 also applies here.

\section{Quadrature formulae error estimates}

Let us divide the interval $[a, b]$ into $v$ subintervals of equal length $h=(b-a) / v$. Assume that $f:[a, b] \rightarrow \mathbf{R}$ is such that $f^{(n-1)}$ is continuous and of bounded variation on $[a, b]$, for some $n \geq 1$. We consider the repeated two-point quadrature formula

$$
\int_{a}^{b} f(t) \mathrm{d} t=M_{\nu}(f)-\sigma_{n-1}(f)+\rho_{n}(f)
$$


and the repeated modified two-point quadrature formula

$$
\int_{a}^{b} f(t) \mathrm{d} t=M_{v}(f)-\sigma_{n}(f)+\tilde{\rho}_{n}(f),
$$

where

$$
\begin{aligned}
M_{\nu}(f) & =\sum_{i=1}^{\nu} M(a+(i-1) h, a+i h) \\
& =\frac{h}{2} \sum_{i=1}^{\nu}[f(a+(i-2 / 3) h)+f(a+(i-1 / 3) h)]
\end{aligned}
$$

and $\sigma_{m}(f)=\sum_{i=1}^{\nu} \tilde{T}_{m}(a+(i-1) h, a+i h), m \geq 0$.

Because of (2.5) we have

$$
\sigma_{0}(f)=\sigma_{1}(f)=0,
$$

while for $m \geq 2$, we get using (4.5) that

$$
\begin{aligned}
\sigma_{m}(f) & =\sum_{i=1}^{\nu} \frac{1}{2} \sum_{j=1}^{[m / 2]} \frac{h^{2 j}}{(2 j) !} \tilde{B}_{2 j}\left[f^{(2 j-1)}(a+i h)-f^{(2 j-1)}(a+(i-1) h)\right] \\
& =\frac{1}{2} \sum_{j=1}^{[m / 2]} \frac{h^{2 j}}{(2 j) !} \tilde{B}_{2 j} \sum_{i=1}^{\nu}\left[f^{(2 j-1)}(a+i h)-f^{(2 j-1)}(a+(i-1) h)\right] \\
& =-\frac{1}{2} \sum_{j=1}^{[m / 2]} \frac{h^{2 j}}{(2 j) !}\left(1-3^{1-2 j}\right) B_{2 j}\left[f^{(2 j-1)}(b)-f^{(2 j-1)}(a)\right]
\end{aligned}
$$

The remainders $\rho_{n}(f)$ and $\tilde{\rho}_{n}(f)$ can be written as

$$
\rho_{n}(f)=\sum_{i=1}^{\nu} \rho_{n}(f ; i), \quad \tilde{\rho}_{n}(f)=\sum_{i=1}^{\nu} \tilde{\rho}_{n}(f ; i),
$$

where, for $i=1, \ldots, \nu$,

$\rho_{n}(f ; i)=\int_{a+(i-1) h}^{a+i h} f(t) \mathrm{d} t-M(a+(i-1) h, a+i h)+\tilde{T}_{n-1}(a+(i-1) h, a+i h)$ and $\tilde{\rho}_{n}(f ; i)=\int_{a+(i-1) h}^{a+i h} f(t) \mathrm{d} t-M(a+(i-1) h, a+i h)+\tilde{T}_{n}(a+(i-1) h, a+i h)$.

We shall apply results from the preceding section to obtain some estimates for the remainders $\rho_{n}(f)$ and $\tilde{\rho}_{n}(f)$. Before doing this, note that for $n=2 k-1(k \geq 2)$, we 
have

$$
\sigma_{2 k-2}(f)=\sigma_{2 k-1}(f)=-\frac{1}{2} \sum_{j=1}^{k-1} \frac{h^{2 j}}{(2 j) !}\left(1-3^{1-2 j}\right) B_{2 j}\left[f^{(2 j-1)}(b)-f^{(2 j-1)}(a)\right] .
$$

Thus $\rho_{2 k-1}(f)=\tilde{\rho}_{2 k-1}(f)$, so that (5.1) and (5.2) coincide in this case. This shows that (5.2) is interesting only when $n=2 k(k \geq 2)$. In this case we have

$$
\begin{aligned}
\tilde{\rho}_{2 k}(f) & =\rho_{2 k}(f)+\sigma_{2 k}(f)-\sigma_{2 k-1}(f) \\
& =\rho_{2 k}(f)-\frac{h^{2 k}}{2[(2 k) !]}\left(1-3^{1-2 k}\right) B_{2 k}\left[f^{(2 k-1)}(b)-f^{(2 k-1)}(a)\right] .
\end{aligned}
$$

In fact we have $\tilde{\rho}_{2 k-2}(f)=\rho_{2 k}(f)(k \geq 2)$.

Therefore for $k \geq 2$ we can approximate $\int_{a}^{b} f(t) \mathrm{d} t$ by

$$
M_{\nu}(f)+\frac{1}{2} \sum_{j=2}^{k-1} \frac{h^{2 j}}{(2 j) !}\left(1-3^{1-2 j}\right) B_{2 j}\left[f^{(2 j-1)}(b)-f^{(2 j-1)}(a)\right],
$$

using either (5.1) with $n=2 k-1$ or (5.2) with $n=2 k-2$. To obtain the error estimate for this approximation, if we apply (5.1), then we must assume that $f^{(2 k-2)}$ is continuous and of bounded variation on $[a, b]$. To do this via (5.2), it is enough to assume that $f^{(2 k-3)}$ is continuous and of bounded variation on $[a, b]$

THEOREM 5. Let $f:[a, b] \rightarrow \mathbf{R}$ be such that $f^{(n-1)}$ is L-Lipschitzian on $[a, b]$ for some $n \geq 1$. For $n=1,2,3,4$ we have, respectively,

$$
\begin{gathered}
\left|\int_{a}^{b} f(t) \mathrm{d} t-M_{\nu}(f)\right| \leq \frac{5}{36} v h^{2} L, \quad\left|\int_{a}^{b} f(t) \mathrm{d} t-M_{\nu}(f)\right| \leq \frac{1}{36} v h^{3} L, \\
\left|\int_{a}^{b} f(t) \mathrm{d} t-M_{\nu}(f)-\frac{h^{2}}{36}\left[f^{\prime}(b)-f^{\prime}(a)\right]\right| \leq \frac{13}{5184} \nu h^{4} L, \\
\left|\int_{a}^{b} f(t) \mathrm{d} t-M_{\nu}(f)-\frac{h^{2}}{36}\left[f^{\prime}(b)-f^{\prime}(a)\right]\right| \leq \frac{13}{19440} v h^{5} L .
\end{gathered}
$$

If $n=2 k-1(k \geq 2)$, then

$$
\begin{aligned}
& \left|\int_{a}^{b} f(t) \mathrm{d} t-M_{\nu}(f)-\frac{1}{2} \sum_{j=1}^{k-1} \frac{h^{2 j}}{(2 j) !}\left(1-3^{1-2 j}\right) B_{2 j}\left[f^{(2 j-1)}(b)-f^{(2 j-1)}(a)\right]\right| \\
& \quad \leq \frac{v h^{2 k}}{(2 k) !} 2\left(1-2^{-2 k}\right)\left(1-3^{1-2 k}\right)\left|B_{2 k}\right| L .
\end{aligned}
$$


If $n=2 k(k \geq 2)$, then

$$
\begin{aligned}
& \left|\int_{a}^{b} f(t) \mathrm{d} t-M_{\nu}(f)-\frac{1}{2} \sum_{j=1}^{k-1} \frac{h^{2 j}}{(2 j) !}\left(1-3^{1-2 j}\right) B_{2 j}\left[f^{(2 j-1)}(b)-f^{(2 j-1)}(a)\right]\right| \\
& \quad \leq \frac{\nu h^{2 k+1}}{2[(2 k) !]}\left(1-3^{1-2 k}\right)\left|B_{2 k}\right| L
\end{aligned}
$$

and

$$
\begin{aligned}
& \left|\int_{a}^{b} f(t) \mathrm{d} t-M_{\nu}(f)-\frac{1}{2} \sum_{j=1}^{k} \frac{h^{2 j}}{(2 j) !}\left(1-3^{1-2 j}\right) B_{2 j}\left[f^{(2 j-1)}(b)-f^{(2 j-1)}(a)\right]\right| \\
& \quad \leq \frac{v h^{2 k+1}}{(2 k) !}\left(1-3^{1-2 k}\right)\left|B_{2 k}\right| L .
\end{aligned}
$$

Proof. Applying (4.1) and (4.2) we get for $i=1, \ldots, \nu$, respectively,

$$
\left|\rho_{n}(f ; i)\right| \leq \frac{h^{n+1}}{2(n !)} \int_{0}^{1}\left|F_{n}(t)\right| \mathrm{d} t L \quad \text { and } \quad\left|\tilde{\rho}_{n}(f ; i)\right| \leq \frac{h^{n+1}}{2(n !)} \int_{0}^{1}\left|G_{n}(t)\right| \mathrm{d} t L .
$$

Using the above estimates and the triangle inequality, we get from (5.5) that

$$
\left|\rho_{n}(f)\right| \leq \sum_{i=1}^{\nu}\left|\rho_{n}(f ; i)\right| \leq \frac{v h^{n+1}}{2(n !)} \int_{0}^{1}\left|F_{n}(t)\right| \mathrm{d} t L
$$

and

$$
\left|\tilde{\rho}_{n}(f)\right| \leq \sum_{i=1}^{\nu}\left|\tilde{\rho}_{n}(f ; i)\right| \leq \frac{v h^{n+1}}{2(n !)} \int_{0}^{1}\left|G_{n}(t)\right| \mathrm{d} t L .
$$

The rest of the argument, from (5.3) and (5.4), is as for Corollaries 3 and 4.

REMARK 9. Instead of the assumption that $f^{(n-1)}$ is $L$-Lipschitzian on $[a, b]$, we can use the stronger assumption that $f^{(n)}$ exists and is bounded on $[a, b]$, for some $n \geq 1$. In this case Theorem 5 applies with $L$ replaced by $\left\|f^{(n)}\right\|_{\infty}$ (see Remark 3 ).

THEOREM 6. Let $f:[a, b] \rightarrow \mathbf{R}$ be such that $f^{(n-1)}$ is continuous and of bounded variation on $[a, b]$ for some $n \geq 1$. For $n=1,2,3,4$ we have, respectively,

$$
\begin{gathered}
\left|\int_{a}^{b} f(t) \mathrm{d} t-M_{\nu}(f)\right| \leq \frac{1}{3} h V_{a}^{b}(f), \quad\left|\int_{a}^{b} f(t) \mathrm{d} t-M_{\nu}(f)\right| \leq \frac{1}{18} h^{2} V_{a}^{b}\left(f^{\prime}\right), \\
\left|\int_{a}^{b} f(t) \mathrm{d} t-M_{\nu}(f)-\frac{h^{2}}{36}\left[f^{\prime}(b)-f^{\prime}(a)\right]\right| \leq \frac{\sqrt{2}}{324} h^{3} V_{a}^{b}\left(f^{\prime \prime}\right), \\
\left|\int_{a}^{b} f(t) \mathrm{d} t-M_{\nu}(f)-\frac{h^{2}}{36}\left[f^{\prime}(b)-f^{\prime}(a)\right]\right| \leq \frac{13}{10368} h^{4} V_{a}^{b}\left(f^{\prime \prime \prime}\right) .
\end{gathered}
$$


If $n=2 k-1(k \geq 2)$, then

$$
\begin{aligned}
& \left|\int_{a}^{b} f(t) \mathrm{d} t-M_{\nu}(f)-\frac{1}{2} \sum_{j=1}^{k-1} \frac{h^{2 j}}{(2 j) !}\left(1-3^{1-2 j}\right) B_{2 j}\left[f^{(2 j-1)}(b)-f^{(2 j-1)}(a)\right]\right| \\
& \quad \leq \frac{h^{2 k-1}}{2[(2 k-1) !]} \max _{t \in[0,1]}\left|F_{2 k-1}(t)\right| V_{a}^{b}\left(f^{(2 k-2)}\right) .
\end{aligned}
$$

If $n=2 k(k \geq 2)$, then

$$
\begin{aligned}
& \left|\int_{a}^{b} f(t) \mathrm{d} t-M_{\nu}(f)-\frac{1}{2} \sum_{j=1}^{k-1} \frac{h^{2 j}}{(2 j) !}\left(1-3^{1-2 j}\right) B_{2 j}\left[f^{(2 j-1)}(b)-f^{(2 j-1)}(a)\right]\right| \\
& \quad \leq \frac{h^{2 k}}{(2 k) !}\left(1-2^{-2 k}\right)\left(1-3^{1-2 k}\right)\left|B_{2 k}\right| V_{a}^{b}\left(f^{(2 k-1)}\right)
\end{aligned}
$$

and

$$
\begin{aligned}
& \left|\int_{a}^{b} f(t) \mathrm{d} t-M_{\nu}(f)-\frac{1}{2} \sum_{j=1}^{k} \frac{h^{2 j}}{(2 j) !}\left(1-3^{1-2 j}\right) B_{2 j}\left[f^{(2 j-1)}(b)-f^{(2 j-1)}(a)\right]\right| \\
& \quad \leq \frac{h^{2 k}}{2[(2 k) !]}\left(1-3^{1-2 k}\right)\left|B_{2 k}\right| V_{a}^{b}\left(f^{(2 k-1)}\right) .
\end{aligned}
$$

Proof. Applying (4.6) and (4.7) we get for $i=1, \ldots, v$ respectively that

$$
\left|\rho_{n}(f ; i)\right| \leq \frac{h^{n}}{2(n !)} \max _{t \in[0,1]}\left|F_{n}(t)\right| V_{a+(i-1) h}^{a+i h}\left(f^{(n-1)}\right)
$$

and

$$
\left|\tilde{\rho}_{n}(f ; i)\right| \leq \frac{h^{n}}{2(n !)} \max _{t \in[0,1]}\left|G_{n}(t)\right| V_{a+(i-1) h}^{a+i h}\left(f^{(n-1)}\right) .
$$

Using the above estimates and the triangle inequality, we get from (5.5) that

$$
\begin{aligned}
\left|\rho_{n}(f)\right| \leq \sum_{i=1}^{\nu}\left|\rho_{n}(f ; i)\right| & \leq \frac{h^{n}}{2(n !)} \max _{t \in[0,1]}\left|F_{n}(t)\right| \cdot \sum_{i=1}^{\nu} V_{a+(i-1) h}^{a+i h}\left(f^{(n-1)}\right) \\
& =\frac{h^{n}}{2(n !)} \max _{t \in[0,1]}\left|F_{n}(t)\right| V_{a}^{b}\left(f^{(n-1)}\right)
\end{aligned}
$$

and similarly $\left|\tilde{\rho}_{n}(f)\right| \leq\left(h^{n} / 2(n !)\right) \max _{t \in[0,1]}\left|G_{n}(t)\right| V_{a}^{b}\left(f^{(n-1)}\right)$. We now use (5.3) and (5.4) and argue as in Corollaries 5 and 6.

REMARK 10. If $f:[a, b] \rightarrow \mathbf{R}$ is such that $f^{(n)} \in L_{1}[a, b]$ for some $n \geq 1$, then $f^{(n-1)}$ is continuous and of bounded variation on $[a, b]$ and $V_{a}^{b}\left(f^{(n-1)}\right)=\left\|f^{(n)}\right\|_{1}$. Therefore Theorem 6 applies with $\left\|f^{(n)}\right\|_{1}$ in place of $V_{a}^{b}\left(f^{(n-1)}\right)$ (see Remark 5). 
THEOREM 7. Assume $(p, q)$ is a pair of conjugate exponents. Let $f:[a, b] \rightarrow \mathbf{R}$ be such that $f^{(n)} \in L_{p}[a, b]$ for some $n \geq 1$. Then $\left|\rho_{n}(f)\right| \leq \nu K(n, p) h^{n+1 / q}\left\|f^{(n)}\right\|_{p}$ and $\left|\tilde{\rho}_{n}(f)\right| \leq \nu K^{*}(n, p) h^{n+1 / q}\left\|f^{(n)}\right\|_{p}$, where $K(n, p)$ and $K^{*}(n, p)$ are defined as in Theorem 4.

Proof. For $i=1, \ldots, v$ let $g_{i}(t)=f^{(n)}(t), t \in[a+(i-1) h, a+i h]$. Then $\left\|g_{i}\right\|_{p} \leq\left\|f^{(n)}\right\|_{p}$, where the norm $\left\|g_{i}\right\|_{p}$ is taken over the interval $[a+(i-1) h, a+i h]$, while the norm $\left\|f^{(n)}\right\|_{p}$ is taken over the interval $[a, b]$. Applying (4.9) and (4.10) and using the above inequality, we get for $i=1, \ldots, v$ that

$$
\left|\rho_{n}(f ; i)\right| \leq K(n, p) h^{n+1 / q}\left\|g_{i}\right\|_{p} \leq K(n, p) h^{n+1 / q}\left\|f^{(n)}\right\|_{p}
$$

and

$$
\left|\tilde{\rho}_{n}(f ; i)\right| \leq K^{*}(n, p) h^{n+1 / q}\left\|g_{i}\right\|_{p} \leq K^{*}(n, p) h^{n+1 / q}\left\|f^{(n)}\right\|_{p}
$$

The result follows from (5.5) by the triangle inequality.

In the following discussion we assume that $f:[a, b] \rightarrow \mathbf{R}$ has a continuous derivative of order $n$, for some $n \geq 1$. In this case we can use (2.4) and the second formula from Remark 1 to obtain, for $i=1, \ldots, v$, that

$$
\begin{aligned}
\rho_{n}(f ; i) & =\frac{h^{n}}{2(n !)} \int_{a+(i-1) h}^{a+i h} F_{n}\left(\frac{t-a-(i-1) h}{h}\right) f^{(n)}(t) \mathrm{d} t \\
& =\frac{h^{n+1}}{2(n !)} \int_{0}^{1} F_{n}(s) f^{(n)}(a+(i-1) h+h s) \mathrm{d} s .
\end{aligned}
$$

Therefore we get by (5.5) that

where

$$
\rho_{n}(f)=\frac{h^{n+1}}{2(n !)} \int_{0}^{1} F_{n}(s) \Phi_{n}(s) \mathrm{d} s,
$$

$$
\Phi_{n}(s)=\sum_{i=1}^{\nu} f^{(n)}(a+(i-1) h+h s), \quad 0 \leq s \leq 1 .
$$

Similarly, we get $\tilde{\rho}_{n}(f)=\left(h^{n+1} / 2(n !)\right) \int_{0}^{1} G_{n}(s) \Phi_{n}(s) \mathrm{d} s$. Obviously, $\Phi_{n}(s)$ is continuous on $[0,1]$ and

$$
\begin{aligned}
\int_{0}^{1} \Phi_{n}(s) \mathrm{d} s & =h^{-1} \sum_{i=1}^{\nu}\left[f^{(n-1)}(a+i h)-f^{(n-1)}(a+(i-1) h)\right] \\
& =h^{-1}\left[f^{(n-1)}(b)-f^{(n-1)}(a)\right] .
\end{aligned}
$$


From the discussion at the beginning of this section, the most interesting case is the repeated tw6-point quadrature formula of Euler type (5.1) for $n=2 k(k \geq 2)$, which can be rewritten as

$$
\begin{aligned}
\int_{a}^{b} f(t) \mathrm{d} t= & M_{\nu}(f)+\frac{1}{2} \sum_{j=1}^{k-1} \frac{h^{2 j}}{(2 j) !}\left(1-3^{1-2 j}\right) B_{2 j}\left[f^{(2 j-1)}(b)-f^{(2 j-1)}(a)\right] \\
& +\rho_{2 k}(f) .
\end{aligned}
$$

The empty sum for $k=1$ is taken as zero.

THEOREM 8. If $f:[a, b] \rightarrow \mathbf{R}$ is such that $f^{(2 k)}$ is continuous on $[a, b]$, for some $k \geq 1$, then there exists a point $\eta \in[a, b]$ such that

$$
\rho_{2 k}(f)=v \frac{h^{2 k+1}}{2[(2 k) !]}\left(1-3^{1-2 k}\right) B_{2 k} f^{(2 k)}(\eta)
$$

PROOF. Using (5.6), we can rewrite $\rho_{2 k}(f)$ as

where

$$
\rho_{2 k}(f)=(-1)^{k-1} \frac{h^{2 k+1}}{2[(2 k) !]} J_{k}
$$

$$
J_{k}=\int_{0}^{1}(-1)^{k-1} F_{2 k}(s) \Phi_{2 k}(s) \mathrm{d} s .
$$

If $m=\min _{t \in[a, b]} f^{(2 k)}(t), M=\max _{t \in[a, b]} f^{(2 k)}(t)$, then we get from (5.7) that $v m \leq \Phi_{2 k}(s) \leq v M, 0 \leq s \leq 1$. On the other hand, (2.8) and Corollary 1 give

$$
(-1)^{k-1} F_{2 k}(s) \geq 0, \quad 0 \leq s \leq 1,
$$

which implies $\nu m \int_{0}^{1}(-1)^{k-1} F_{2 k}(s) \mathrm{d} s \leq J_{k} \leq \nu M \int_{0}^{1}(-1)^{k-1} F_{2 k}(s) \mathrm{d} s$. Using (3.10) we have $\nu m(-1)^{k} \tilde{B}_{2 k} \leq J_{k} \leq \nu M(-1)^{k} \tilde{B}_{2 k}$. By the continuity of $f^{(2 k)}(s)$ on $[a, b]$, it follows that there must exist a point $\eta \in[a, b]$ such that $J_{k}=v(-1)^{k} \tilde{B}_{2 k} f^{(2 k)}(\eta)$. Combining this with (5.11) and (3.3) gives (5.10).

REMARK 11. The repeated two-point quadrature formula of Euler type (5.9) is a generalisation of the two-point formula (1.2). Namely, from (5.10) for $k=1$ and $\nu=1$ we get $\rho_{2}(f)=\left((b-a)^{3} / 36\right) f^{\prime \prime}(\eta)$ and (5.9) reduces to (1.2).

THEOREM 9. If $f:[a, b] \rightarrow \mathbf{R}$ is such that $f^{(2 k)}$ is continuous on $[a, b]$, for some $k \geq 1$, and does not change sign on $[a, b]$, then there exists a point $\theta \in[0,1]$ such that

$$
\rho_{2 k}(f)=\theta \frac{h^{2 k}}{(2 k) !}\left(1-2^{-2 k}\right)\left(1-3^{1-2 k}\right) B_{2 k}\left[f^{(2 k-1)}(b)-f^{(2 k-1)}(a)\right]
$$


Proof. Suppose that $f^{(2 k)}(t) \geq 0, a \leq t \leq b$. Then from (5.7) we get $\Phi_{2 k}(s) \geq 0$, $0 \leq s \leq 1$. It follows from Corollary 1 that $0 \leq(-1)^{k-1} F_{2 k}(s) \leq(-1)^{k-1} F_{2 k}(1 / 2)$, $0 \leq s \leq 1$. Therefore if $J_{k}$ is given by (5.12), $0 \leq J_{k} \leq(-1)^{k-1} F_{2 k}(1 / 2) \int_{0}^{1} \Phi_{2 k}(s) \mathrm{d} s$.

Using (5.8), we get

$$
0 \leq J_{k} \leq(-1)^{k-1} 2\left(1-2^{-2 k}\right)\left(1-3^{1-2 k}\right) B_{2 k} h^{-1}\left[f^{(2 k-1)}(b)-f^{(2 k-1)}(a)\right],
$$

which means that there must exist a point $\theta \in[0,1]$ such that

$$
J_{k}=\theta(-1)^{k-1} 2\left(1-2^{-2 k}\right)\left(1-3^{1-2 k}\right) B_{2 k} h^{-1}\left[f^{(2 k-1)}(b)-f^{(2 k-1)}(a)\right] .
$$

Combining this with (5.11) gives (5.13). When $f^{(2 k)}(t) \leq 0(a \leq t \leq b)$ the argument is the same, since in that case we get

$$
(-1)^{k-1} 2\left(1-2^{-2 k}\right)\left(1-3^{1-2 k}\right) B_{2 k} h^{-1}\left[f^{(2 k-1)}(b)-f^{(2 k-1)}(a)\right] \leq J_{k} \leq 0 .
$$

REMARK 12. If we approximate $\int_{a}^{b} f(t) d t$ by

$$
I_{2 k}(f)=M_{\nu}(f)+\frac{1}{2} \sum_{j=1}^{k-1} \frac{h^{2 j}}{(2 j) !}\left(1-3^{1-2 j}\right) B_{2 j}\left[f^{(2 j-1)}(b)-f^{(2 j-1)}(a)\right],
$$

then the next approximation will be $I_{2 k+2}(f)$. The difference $\Delta_{2 k}(f):=I_{2 k+2}(f)-$ $I_{2 k}(f)$ is equal to the last term in the sum in $I_{2 k+2}(f)$, that is,

$$
\Delta_{2 k}(f)=\frac{h^{2 k}}{2[(2 k) !]}\left(1-3^{1-2 k}\right) B_{2 k}\left[f^{(2 k-1)}(b)-f^{(2 k-1)}(a)\right] .
$$

We see that, under the assumptions of Theorem $9, \rho_{2 k}(f)$ and $\Delta_{2 k}(f)$ are of the same sign. Moreover, we have $\rho_{2 k}(f)=2 \theta\left(1-2^{-2 k}\right) \Delta_{2 k}(f)$, which yields the simple estimate $\left|\rho_{2 k}(f)\right| \leq 2\left|\Delta_{2 k}(f)\right|$ for the remainder $\rho_{2 k}(f)$.

THEOREM 10. Suppose that $f:[a, b] \rightarrow \mathbf{R}$ is such that $f^{(2 k+2)}$ is continuous on $[a, b]$, for some $k \geq 1$. If for each $x \in[a, b], f^{(2 k)}(x)$ and $f^{(2 k+2)}(x)$ are either both nonnegative or both nonpositive, then the remainder $\rho_{2 k}(f)$ has the same sign as the first neglected term $\Delta_{2 k}(f)$ given by (5.14). Moreover, we have the estimate $\left|\rho_{2 k}(f)\right| \leq\left|\Delta_{2 k}(f)\right|$.

ProOF. We have $\Delta_{2 k}(f)+\rho_{2 k+2}(f)=\rho_{2 k}(f)$, that is,

$$
\Delta_{2 k}(f)=-\rho_{2 k+2}(f)+\rho_{2 k}(f) .
$$

By (5.6)

$$
-\rho_{2 k+2}(f)=\frac{h^{2 k+3}}{2[(2 k+2) !]} \int_{0}^{1}\left[-F_{2 k+2}(s)\right] \Phi_{2 k+2}(s) \mathrm{d} s
$$


and

$$
\rho_{2 k}(f)=\frac{h^{2 k+1}}{2[(2 k) !} \int_{0}^{1} F_{2 k+2}(s) \Phi_{2 k}(s) \mathrm{d} s .
$$

Under the assumptions made on $f$ we see that for all $s \in[0,1], \Phi_{2 k}(s)$ and $\Phi_{2 k+2}(s)$ are either both nonnegative or both nonpositive. Also, from (2.8) and Corollary 1 it follows that for all $s \in[0,1],(-1)^{k-1}\left[-F_{2 k+2}(s)\right] \geq 0$ and $(-1)^{k-1} F_{2 k}(s) \geq 0$.

We conclude that $-\rho_{2 k+2}(f)$ and $\rho_{2 k}(f)$ have the same sign. Because of (5.15), $\Delta_{2 k}(f)$ must therefore have the same sign as $-\rho_{2 k+2}(f)$ and $\rho_{2 k}(f)$. Moreover, it follows that $\left|-\rho_{2 k+2}(f)\right| \leq\left|\Delta_{2 k}(f)\right|$ and $\left|\rho_{2 k}(f) \leq\right| \Delta_{2 k}(f) \mid$.

\section{References}

[1] M. Abramowitz and I. A. Stegun (eds.), Handbook of mathematical functions with formulae, graphs and mathematical tables, Appl. Math. Series 55, 4th printing (National Bureau of Standards, Washington, 1965).

[2] I. S. Berezin and N. P. Zhidkov, Computing methods. Vol. I (Pergamon Press, Oxford, 1965).

[3] P. J. Davis and P. Rabinowitz, Methods of numerical integration (Academic Press, New York, 1975).

[4] Lj. Dedić, M. Matić and J. Pečarič, "On Euler midpoint formulae", ANZIAM J. , to appear.

[5] Lj. Dedić, M. Matić and J. Pečarić, “On generalizations of Ostrowski inequality via some Euler-type identities", Math. Inequal. Appl. 3 (2000) 337-353.

[6] S. S. Dragomir, "On Simpson's quadrature formula for differentiable mappings whose derivatives belong to $L_{p}$ spaces and applications", J. KSIAM 22 (1998) 57-65.

[7] S. S. Dragomir, "On Simpson's quadrature formula for Lipschitzian mappings and applications", Soochow J. Math. 25 (1999) 175-180.

[8] S. S. Dragomir, "On Simpson's quadrature formula for mappings of bounded variation and applications", Tamkang J. Math. 30 (1999) 53-58.

[9] S. S. Dragomir, R. P. Agarwal and P. Cerone, “On Simpson's inequality and applications”, J. Inequal. Appl. 5 (2000) 533-579.

[10] V. 1. Krylov, Approximate calculation of integrals (Macmillan, New York, 1962).

[11] J. E. Pečarić, F. Proschan and Y. L. Tong, Convex functions, partial orderings and statistical applications (Academic Press, Boston, 1992). 